EUROPEAN JOURNAL OF PURE AND APPLIED MATHEMATICS

Vol. 13, No. 3, 2020, 390-402

ISSN 1307-5543 - www.ejpam.com

Published by New York Business Global

\title{
E-J Summability of Orthogonal Series
}

F. Aydin Akgun $^{1, *}$, B. E. Rhoades ${ }^{2}$

1 Department of Mathematical Engineering, Yildiz Technical University, 34210 Esenler, Istanbul, Turkey

${ }^{2}$ Department of Mathematics, Indiana University, Bloomington, IN 47405-7106, U.S.A.

\begin{abstract}
In this paper we obtain a sufficient condition for the E-J summability of certain orthogonal series. Our results generalize the corresponding theorems for ordinary Hausdorff summability obtained by Kalaivana and Youvaraj.
\end{abstract}

2020 Mathematics Subject Classifications: 40G05

Key Words and Phrases: E-J matrices, orthogonal series

\section{Preliminaries}

The set of all real or complex sequences $\left\{x_{n}\right\}$ for which $A_{n}(x):=\sum_{k} a_{n k} x_{k}$ converges is called the convergence domain of $A$, written $c_{A}$, where $A$ is an infinite matrix. A matrix $A$ is said to be conservative if it maps each convergent sequence into a convergent sequence, not necessarily with the same limit. If the limit is also preserved, then the matrix is called regular. Silverman and Toeplitz established necessary and sufficient conditions for a matrix to be conservative[4]. They are

(i) $\|A\|_{\infty}:=\sup _{n} \sum_{k}\left|a_{n k}\right|<\infty$,

(ii) $t:=\lim _{n} \sum_{k} a_{n k}$ exists,

(iii) $a_{k}:=\lim _{n} a_{n k}$ exists for each $k$.

A Hausdorff matrix $H=\left(h_{n k}\right)$ is a lower triangular matrix with nonzero entries

$$
h_{n k}=\left(\begin{array}{c}
n \\
k
\end{array}\right) \Delta^{n-k} \mu_{k}
$$

where $\left\{\mu_{n}\right\}$ is any real sequence and $\Delta$ is the forward difference operator defied by $\Delta \mu_{k}=$ $\mu_{k}-\mu_{k+1}$ and $\Delta^{n+1} \mu_{k}=\Delta\left(\Delta^{n} \mu_{k}\right)$. For every Hausdorff matrix each row sum is equal to $\mu_{0}[3]$.

*Corresponding author.

DOI: https://doi.org/10.29020/nybg.ejpam.v13i3.3687

Email addresses: fakgun@yildiz.edu.tr (F. Aydin Akgun), rhoades@indiana.edu (B. E. Rhoades) 
F. Hausdorff [2] proved that a Hausdorff matrix is conservative if and only if

$$
\mu_{n}=\int_{0}^{1} x^{n} d_{\chi}(x)
$$

where the mass function $\chi \in B V[0,1]$.

The E-J generalized Hausdorff matrices, denoted by $H_{\mu}^{\alpha}=\left(h_{n k}^{(\alpha)}\right)$, were defined independently by Endl [1] and Jakimovski [5], with nonzero entries

$$
h_{n k}^{(\alpha)}=\left(\begin{array}{c}
n+\alpha \\
n-k
\end{array}\right) \Delta^{n-k} \mu_{k}^{(\alpha)}, 0 \leq k \leq n,
$$

for any $\alpha \geq 0$. For $\alpha=0$, the E-J matrices reduce to the ordinary Hausdorff matrices.

If the $\mu_{n}^{(\alpha)}$ satisfy the condition

$$
\mu_{n}^{(\alpha)}=\int_{0}^{1} x^{n+\alpha} d \chi(x)
$$

where $\chi \in B V[0,1]$, then the corresponding E-J matrix is conservative.

Definition 1. Let $\gamma:[1, \infty) \rightarrow[0, \infty)$ be a nondecreasing function, $A=\left(a_{n k}\right)$ an infinite matrix. Then a series $\sum_{n} b_{n}$ is said to be $|A, \gamma|_{k}$ summable, if

$$
\sum_{n=1}^{\infty} \gamma(n)^{k} n^{k-1}\left|\sigma_{n}-\sigma_{n-1}\right|^{k}
$$

converges, where $\sigma_{n}: \sum_{n} a_{n k} b_{k}$.

Definition 2. Let $\gamma:=\left\{\gamma_{n}\right\}$ be a positive sequence, $\beta$ a real positive number. Then $\gamma$ is called quasi- $\beta$-power monotone decreasing if there exists a number $M=M(\beta, \gamma) \geq 1$ such that

$$
n^{\beta} \gamma(n) \leq M m^{\beta} \gamma(m)
$$

for each $m \leq n$.

For any real number $\beta, \Gamma_{\beta}$ denotes the set of all increasing functions $\Gamma_{\beta}:[1, \infty) \rightarrow$ $[0, \infty)$ such that each $\left\{\gamma_{n}\right\}$ is a quasi $\beta$-power monotone decreasing sequence.

\section{Main Results}

Theorem 1. Let $\left\{\varphi_{n}\right\}_{n=0}^{\infty} \subset E_{2}[0,1]$ be an orthonormal system, $H_{\mu}^{\alpha}$ an E-J Hausdorff matrix with $\chi$ monotone decreasing, $\gamma \in \Gamma_{\beta}$ for $\beta>1-1 / k, 1 \leq k \leq 2$. Then every orthogonal series $\sum_{n=0}^{\infty} b_{n} \varphi_{n}$ is $\left|H^{\alpha}, \gamma\right|$ summable.

The following lemmas will be needed in the proof of Theorem 1 . 
Lemma 1. Let $H^{\alpha}$ be an E-J matrix with entries $\left(h_{n k}\right)$, where $\chi$ is a monotonically increasing mass function on $[0,1]$ associated with the $\mu_{n}$. Then

(i) $a_{m n}=K\left(\begin{array}{c}n-1+\alpha \\ m-1+\alpha\end{array}\right) \xi^{m+\alpha}(1-\xi)^{n-m}$ for some $\xi \in(0,1)$,

(ii) $\sum_{m=0}^{n}\left|a_{m n}\right|^{2}\left|b_{m}\right|^{2} \leq K^{2} \sum_{m=0}^{n}\left|b_{m}\right|^{2}$ for all $b_{n} \in \mathbb{C}$ and $n \in \mathbb{N}$, where $K=\chi(1)-\chi(0)$ and $a_{m n}=\sum_{k=m}^{n}\left|h_{n k}^{(\alpha)}-h_{n-1, k}^{(\alpha)}\right|$.

Here $\mathbb{C}=$ complex numbers and $\mathbb{N}=$ natural numbers.

Proof. (i) We consider

$$
\begin{gathered}
h_{n k}^{(\alpha)}-h_{n-1, k}^{(\alpha)} \\
=\left[\int_{0}^{1} \mu^{k+\alpha}(1-\mu)^{n-k}\left(\begin{array}{l}
n+\alpha \\
k+\alpha
\end{array}\right) d \chi(\mu)-\int_{0}^{1} \mu^{k+\alpha}(1-\mu)^{n-1-k}\left(\begin{array}{c}
n-1+\alpha \\
k+\alpha
\end{array}\right) d \chi(\mu)\right] \\
=\int_{0}^{1} \mu^{k+\alpha}(1-\mu)^{n-k}\left[\left(\begin{array}{c}
n+\alpha \\
k+\alpha
\end{array}\right)-\left(\begin{array}{c}
n-1+\alpha \\
k+\alpha
\end{array}\right) \frac{1}{1-\mu}\right] d \chi(\mu),
\end{gathered}
$$

where $0 \leq k \leq n$. Since

$$
\left(\begin{array}{l}
n+\alpha \\
k+\alpha
\end{array}\right)-\left(\begin{array}{c}
n-1+\alpha \\
k+\alpha
\end{array}\right)=\left(\begin{array}{l}
n-1+\alpha \\
k-1+\alpha
\end{array}\right)
$$

from (2),

$$
\begin{aligned}
& \int_{0}^{1} \mu^{k+\alpha}(1-\mu)^{n-1-k}\left[\left(\begin{array}{c}
n+\alpha \\
k+\alpha
\end{array}\right)(1-\mu)-\left(\begin{array}{c}
n-1+\alpha \\
k+\alpha
\end{array}\right)\right] d \chi(\mu) \\
& =\int_{0}^{1} \mu^{k+\alpha}(1-\mu)^{n-1-k}\left[\left(\begin{array}{c}
n+\alpha \\
k+\alpha
\end{array}\right)-\left(\begin{array}{c}
n-1+\alpha \\
k+\alpha
\end{array}\right)-\mu\left(\begin{array}{l}
n+\alpha \\
k+\alpha
\end{array}\right)\right] d \chi(\mu) \\
& =\int_{0}^{1} \mu^{k+\alpha}(1-\mu)^{n-1-k}\left[\left(\begin{array}{c}
n-1+\alpha \\
k-1+\alpha
\end{array}\right)-\mu\left(\begin{array}{l}
n+\alpha \\
k+\alpha
\end{array}\right)\right] d \chi(\mu) .
\end{aligned}
$$

Thus

$$
\begin{aligned}
a_{m n}=\sum_{k=m}^{n}\left(h_{n k}^{(\alpha)}-h_{n-1, k}^{(\alpha)}\right) & =\sum_{k=m}^{n} \int_{0}^{1} \mu^{k+\alpha}(1-\mu)^{n-1-k}\left[\left(\begin{array}{l}
n-1+\alpha \\
k-1+\alpha
\end{array}\right)-\mu\left(\begin{array}{l}
n+\alpha \\
k+\alpha
\end{array}\right)\right] d \chi(\mu) \\
& =\int_{0}^{1} \sum_{k=m}^{n} \mu^{k+\alpha}(1-\mu)^{n-1-k}\left[\left(\begin{array}{l}
n-1+\alpha \\
k-1+\alpha
\end{array}\right)-\mu\left(\begin{array}{l}
n+\alpha \\
k+\alpha
\end{array}\right)\right] d \chi(\mu) .
\end{aligned}
$$

From the above inequality,

$$
\sum_{k=m}^{n} \mu^{k+\alpha}(1-\mu)^{n-1-k}\left[\left(\begin{array}{l}
n-1+\alpha \\
k-1+\alpha
\end{array}\right)-\mu\left(\begin{array}{l}
n+\alpha \\
k+\alpha
\end{array}\right)\right]
$$




$$
\begin{aligned}
= & \sum_{k=m}^{n} \mu^{k+\alpha}(1-\mu)^{n-1-k}\left(\begin{array}{l}
n-1+\alpha \\
k-1+\alpha
\end{array}\right)-\sum_{k=m}^{n} \mu^{k+\alpha+1}(1-\mu)^{n-1-k}\left(\begin{array}{l}
n+\alpha \\
k+\alpha
\end{array}\right) \\
= & \sum_{k=m}^{n} \mu^{k+\alpha}(1-\mu)^{n-1-k}\left(\begin{array}{l}
n-1+\alpha \\
k-1+\alpha
\end{array}\right)-\sum_{k=m}^{n} \mu^{k+\alpha+1}(1-\mu)^{n-1-k}\left[\left(\begin{array}{c}
n-1+\alpha \\
k-1+\alpha
\end{array}\right)+\left(\begin{array}{c}
n-1+\alpha \\
k+\alpha
\end{array}\right)\right] \\
= & \sum_{k=m}^{n} \mu^{k+\alpha}(1-\mu)^{n-k}\left(\begin{array}{c}
n-1+\alpha \\
k-1+\alpha
\end{array}\right)-\sum_{k=m}^{n} \mu^{k+\alpha+1}(1-\mu)^{n-1-k}\left(\begin{array}{c}
n-1+\alpha \\
k+\alpha
\end{array}\right) \\
= & \sum_{k=m}^{n} \mu^{k+\alpha}(1-\mu)^{n-k}\left(\begin{array}{c}
n-1+\alpha \\
k-1+\alpha
\end{array}\right)-\sum_{g=m+1}^{n+1} \mu^{g+\alpha}(1-\mu)^{n-g}\left(\begin{array}{c}
n-1+\alpha \\
g-1+\alpha
\end{array}\right) \\
= & \mu^{m+\alpha}(1-\mu)^{n-m}\left(\begin{array}{c}
n-1+\alpha \\
m-1+\alpha
\end{array}\right),
\end{aligned}
$$

and

$$
0 \leq a_{m n} \leq \int_{0}^{1} \mu^{m+\alpha}(1-\mu)^{n-m}\left(\begin{array}{c}
n-1+\alpha \\
m-1+\alpha
\end{array}\right) d \chi(\mu) .
$$

Using the first mean value theorem for integrals, for some $0<\xi<1$,

$$
\begin{aligned}
\int_{0}^{1} \mu^{m+\alpha}(1-\mu)^{n-m}\left(\begin{array}{c}
n-1+\alpha \\
m-1+\alpha
\end{array}\right) d \chi(\mu) & =\xi^{m+\alpha}(1-\xi)^{n-m}\left(\begin{array}{c}
n-1+\alpha \\
m-1+\alpha
\end{array}\right) \int_{0}^{1} d \chi(\mu) \\
& =K \xi^{m+\alpha}(1-\xi)^{n-m}\left(\begin{array}{c}
n-1+\alpha \\
m-1+\alpha
\end{array}\right),
\end{aligned}
$$

where $0<K \leq 1$, and (i) is satisfied.

To prove (ii) we need the following lemma.

Lemma 2. For $0<K<1, a_{m n} \leq 1$.

Proof. From (3)

$$
\begin{aligned}
a_{m n} & =K \int_{0}^{1} \mu^{m+\alpha}(1-\mu)^{n-m}\left(\begin{array}{c}
n-1+\alpha \\
m-1+\alpha
\end{array}\right) d \mu \\
& =K\left(\begin{array}{c}
n-1+\alpha \\
m-1+\alpha
\end{array}\right) \int_{0}^{1} \mu^{m+\alpha}(1-\mu)^{n-m} d \mu \\
& =K\left(\begin{array}{c}
n-1+\alpha \\
m-1+\alpha
\end{array}\right) \frac{\Gamma(m+\alpha+1) \Gamma(n-m+1)}{\Gamma(n+\alpha+2)} \\
& =K \frac{\Gamma(n+\alpha)}{\Gamma(m+\alpha) \Gamma(n-m+1)} \frac{\Gamma(m+\alpha+1) \Gamma(n-m+1)}{\Gamma(n+\alpha+2)} \\
& =K \frac{(m+\alpha)}{(n+\alpha+1)(n+\alpha)} .
\end{aligned}
$$

Since $n \geq m$,

$$
a_{m n} \leq K<1 .
$$


Using equation (4) we can write

$$
\sum_{m=0}^{n}\left|a_{m n}\right|^{2}\left|b_{m}\right|^{2} \leq K^{2} \sum_{m=0}^{n}\left|b_{m}\right|^{2} \leq \sum_{m=0}^{n}\left|b_{m}\right|^{2}
$$

which is a proof of (ii).

Lemma 3. Let $\left\{\varphi_{n}\right\}_{n=0}^{\infty} \subset L_{2}[0,1]$ be an orthonormal system, $H_{\mu}^{\alpha}$ be an E-J Hausdorff matrix with monotonically increasing function $\chi$ on $[0,1]$. Then, for $n \in \mathbb{N}$ and

$$
K=\int_{0}^{1} d \chi(\mu)
$$

(i) there exists $\xi \in(0,1)$ such that

$$
\int_{0}^{1}\left|\sigma_{n}(x)-\sigma_{n-1}(x)\right|^{2}=K^{2} \sum_{m=0}^{n} \xi^{2 m+2 \alpha}(1-\xi)^{2 n-2 m}\left(\begin{array}{c}
n-1+\alpha \\
m-1+\alpha
\end{array}\right)^{2}\left|b_{m}\right|^{2}
$$

and

$$
\int_{0}^{1}\left|\sigma_{n}(x)-\sigma_{n-1}(x)\right|^{2} d x=K^{2} \sum_{m=0}^{n}\left|b_{m}\right|^{2}
$$

for all $b_{m} \in \mathbb{C}$ where, for $n \in \mathbb{N}, \sigma_{n}(x)=\sum_{k=0}^{n} h_{n k} S_{k}(x)$, where $S_{k}$ denotes the $k^{\text {th }}$ partial sum of the orthogonal series $\sum_{m=0}^{\infty} b_{m} \varphi_{m}$.

Proof.

$$
\begin{aligned}
& \sigma_{n}(x)-\sigma_{n-1}(x)=\sum_{k=0}^{n}\left(h_{n k}^{(\alpha)}-h_{n-1, k}^{(\alpha)}\right) S_{k}(x) \\
& =\sum_{k=0}^{n}\left(h_{n k}^{(\alpha)}-h_{n-1, k}^{(\alpha)}\right) \sum_{m=0}^{k} b_{m} \varphi_{m} \\
& =\sum_{m=0}^{n} \sum_{k=m}^{n}\left(h_{n k}^{(\alpha)}-h_{n-1, k}^{(\alpha)}\right) b_{m} \varphi_{m} \\
& =\sum_{m=0}^{n} a_{m n} b_{m} \varphi_{m} .
\end{aligned}
$$

Since $\left\{\varphi_{n}\right\}_{n=0}^{\infty}$ is an orthonormal system, using Parseval's identity,

$$
\begin{aligned}
\int_{0}^{1}\left|\sigma_{n}(x)-\sigma_{n-1}(x)\right|^{2} d x & =\sum_{m=0}^{n}\left|a_{m n}\right|^{2}\left|b_{m}\right|^{2} \\
& =K^{2} \sum_{m=0}^{n} \xi^{2 m+2 \alpha}(1-\xi)^{2 n-2 m}\left(\begin{array}{c}
n-1+\alpha \\
m-1+\alpha
\end{array}\right)^{2}\left|b_{m}\right|^{2}
\end{aligned}
$$


From Lemma 2,

$$
\int_{0}^{1}\left|\sigma_{n}(x)-\sigma_{n-1}(x)\right|^{2} d x=\sum_{m=0}^{n}\left|a_{m n}\right|^{2}\left|b_{m}\right|^{2} \leq K^{2} \sum_{m=0}^{n}\left|b_{m}\right|^{2} .
$$

Proof. To prove Theorem 1, from Definition 1 we need to show that

$$
\sum_{n=1}^{\infty} \gamma(n)^{k} n^{k-1}\left|\sigma_{n}-\sigma_{n-1}\right|^{k}
$$

converges for $1 \leq k<2$, where, for $n \in \mathbb{N}, \sigma_{n}(x) \sum_{k=0}^{n} h_{n k}^{(\alpha)} S_{k}(x)$.

Using Lemma 3, and Hölder's inequality with $p=2 / k$, for any $1 \leq k \leq 2$, and for all $b \in \ell_{2}\left(\mathbb{Z}^{+}\right)$, we have

$$
\begin{aligned}
\sum_{n=1}^{\infty} \gamma(n)^{k} n^{k} \int_{0}^{n}\left|\sigma_{n}(x)-\sigma_{n-1}(x)\right|^{k} d x & \leq \sum_{n=1}^{\infty} \gamma(n)^{k} n^{k-1}\left\{K^{2}\|b\|_{2}^{2}\right\}^{k / 2} \\
& \leq\left\{K\|b\|_{2}\right\}^{k} \sum_{n=1}^{\infty} \gamma(n)^{k} n^{k-1}
\end{aligned}
$$

Here $\{\gamma(n)\}$ is a quasi $\beta$-power monotone decreasing sequence with $\beta>1-1 / k$, and, since for $\epsilon=\beta-1+1 / k$, the sequence $\left\{n^{k-1} \gamma(n)^{k}\right\}$ is quasi $k \epsilon$-power monotone decreasing. Using Lemma 1 of $[6]$, we have

$$
\begin{aligned}
& \leq\left\{K\|b\|_{2}\right\}^{k} \sum_{n=1}^{\infty} \gamma\left(2^{n}\right)^{k}\left(2^{n}\right)^{k-1} \\
& \leq\left\{K\|b\|_{2}\right\}^{k} B \gamma(2)^{k}(2)^{k-1}
\end{aligned}
$$

where $B \geq 1$.

Theorem 2. Let $\{\varphi\}_{n=0}^{\infty} \subset L_{2}[0,1]$ be an orthogonal system and $H_{\mu}^{\alpha}$ the corresponding E-J Hausdorff matrix. For $1 \leq k \leq 2$ and $\gamma \in \Gamma(\beta)$ with $\beta>1-1 / k$, every orthogonal series $\sum_{n=0}^{\infty} b_{n} \varphi_{n}$ is $\left|H^{\alpha}, \gamma\right|_{k}$ summable.

Proof. Let $\chi \in B V[0,1]$ be the mass function corresponding to the E-J matrix $H^{\alpha}$. By the Jordan decomposition theorem, $\chi=\chi_{1}-\chi_{2}$, where $\chi_{1}$ and $\chi_{2}$ are monotone increasing functions. To prove the theorem we apply Theorem 1 to $\chi_{1}$ and $\chi_{2}$. Theorems 1 and 2 are generalizations of Theorems 1 and 2, respectively, in [6].

Theorem 3. Let $\{\varphi\}_{n=0}^{\infty} \subset L_{2}[0,1]$ be an orthogonal system and $H_{\mu}^{\alpha}$ an E-J Hausdorff matrix with $\chi \in[0,1]$ and monotone increasing. For $1 \leq k \leq 2$ and $\gamma \in \Gamma(\beta)$ with $\beta>1-1 / k$, a sufficient condition for the orthogonal series $\sum_{n=0}^{\infty} b_{n} \varphi_{n}$ to be $\left|H^{\alpha}, \gamma\right|_{k}$ summable is

$$
\sum_{s=0}^{\infty} \gamma\left(2^{s}\right)^{k}\left\{\sum_{m=2^{s}+1}^{2^{s+1}} \sqrt{m+\alpha}\left|b_{m}\right|^{2}\right\}^{k / 2}<\infty .
$$


Proof. Let $\chi \in B V[0,1]$ and monotonically increasing on $[0,1]$. By Lemma 1(i) there exists a $\xi \in(0,1)$ such that

$$
\int_{0}^{1}\left|\sigma_{n}(x)-\sigma_{n-1}(x)\right| d x \leq K^{2} \sum_{m=0}^{n}\left(\begin{array}{c}
n+\alpha-1 \\
m+\alpha-1
\end{array}\right)^{2} \xi^{2 m+2 \alpha}(1-\xi)^{2 n-2 m}\left|b_{m}\right|^{2},
$$

where

$$
\sigma_{n}(x)=\sum_{k=0}^{n} h_{n k}^{(\alpha)} S_{k}
$$

For $1 \leq k \leq 2$, by using Hölder's inequality and equation (1),

$$
\begin{gathered}
\sum_{n=2}^{\infty} \gamma(n)^{k} n^{k-1}\left\{\int_{0}^{1}\left|\sigma_{n}(x)-\sigma_{n-1}(x)\right| d x\right\}^{k} \\
\leq \sum_{n=2}^{\infty} \gamma(n)^{k} n^{k-1}\left\{K^{2} \sum_{m=0}^{n}\left(\begin{array}{c}
n+\alpha-1 \\
m+\alpha-1
\end{array}\right)^{2} \xi^{2 m+2 \alpha}(1-\xi)^{2 n-2 m}\left|b_{m}\right|^{2}\right\}^{k / 2} .
\end{gathered}
$$

Replacing $\xi$ by $1 /(1+q)$ in (8), we obtain

$$
=K^{k} \sum_{r=0}^{\infty} \sum_{n=2^{r}+1}^{2^{r+1}} \gamma(n)^{k} n^{k-1}\left\{\sum_{n=0}^{n}\left(\begin{array}{c}
n+\alpha \\
m+\alpha
\end{array}\right)^{2}\left(\frac{m+\alpha}{n+\alpha}\right)^{2} q^{2 n-2 m}(1+q)^{-2 n-2 \alpha}\left|b_{m}\right|^{2}\right\}^{k / 2}
$$

O.A. Ziza [7] proved that, for $q>0$, there exists a constant $C_{q}>0$ such that

$$
\max _{0 \leq k \leq n}\left(\begin{array}{l}
n \\
k
\end{array}\right) q^{k} \leq C_{q} \frac{(1+q)^{n}}{\sqrt{n}}, \quad n=1,2, \ldots .
$$

We shall generalize this Lemma for E-J matrices.

Lemma 4. For $q>0$ there exists a $C_{q}>0$ such that

$$
\max _{0 \leq k \leq n}\left(\begin{array}{l}
n+\alpha \\
k+\alpha
\end{array}\right) q^{k+\alpha} \leq C_{q} \frac{(1+q)^{n+\alpha}}{\sqrt{n+\alpha}}, \quad n=1,2, \ldots
$$

Proof.

$$
\frac{\left(\begin{array}{c}
n+\alpha+1 \\
k+\alpha
\end{array}\right)}{\left(\begin{array}{c}
n+\alpha \\
k+\alpha-1
\end{array}\right)}=\frac{n+\alpha+1}{k+\alpha}
$$

Let

$$
d_{k}=\left(\frac{n+\alpha+1}{k+\alpha}-1\right) q .
$$

The $d_{n}$ are decreasing in $k$. Let $k_{n}$ denote the largest value of $k+\alpha$ for which $d_{k_{n}} \geq 1$. Then $d_{k_{n+1}}<1$, and

$$
\max _{0 \leq k \leq n}\left(\begin{array}{c}
n+\alpha \\
k+\alpha
\end{array}\right) q^{k+\alpha}=\left(\begin{array}{c}
n+\alpha \\
k_{n}
\end{array}\right) q^{k_{n}}
$$


It then follows that one can write

$$
k_{n}=\frac{q}{1+q}(n+\alpha)+\nu_{n}
$$

where $0<\nu_{n}<1$.

Then

$$
\left(\begin{array}{c}
n+\alpha \\
k_{n}
\end{array}\right) \leq C_{1} \frac{(n+\alpha) !}{k_{n} !\left(n+\alpha-k_{n}\right) !}=\frac{(n+\alpha)^{n+\alpha} e^{-(n+\alpha)} \sqrt{n+\alpha}}{\left(k_{n}\right)^{k_{n}} e^{-\left(k_{n}\right)} \sqrt{k_{n}}\left(n+\alpha-k_{n}\right)^{\left(n+\alpha-k_{n}\right)} e^{-\left(n+\alpha-k_{n}\right)} \sqrt{n+\alpha-k_{n}}} .
$$

With $p=q /(1+q)$, the right hand side of (10) equals

$$
\begin{gathered}
\frac{(n+\alpha)^{(n+\alpha)} e^{-(n+\alpha)} \sqrt{n+\alpha}}{\left(p(n+\alpha)+\nu_{n}\right)^{\left(p(n+\alpha)+\nu_{n}\right)} e^{-\left(p(n+\alpha)+\nu_{n}\right)} \sqrt{\left(p(n+\alpha)+\nu_{n}\right)}} \\
\times \frac{1}{\left(n+\alpha-p(n+\alpha)-\nu_{n}\right)^{\left(n+\alpha-p(n+\alpha)-\nu_{n}\right)} e^{-\left(n+\alpha-p(n+\alpha)-\nu_{n}\right)} \sqrt{\left(n+\alpha-p(n+\alpha)-\nu_{n}\right)}} \\
=\frac{(n+\alpha)^{p(n+\alpha)+\nu_{n}}}{\left(p(n+\alpha)+\nu_{n}\right)^{\left(p(n+\alpha)+\nu_{n}\right)}} \times \frac{(n+\alpha)^{n+\alpha-p(n+\alpha)-\nu_{n}}}{\left(n+\alpha-p(n+\alpha)-\nu_{n}\right)^{n+\alpha-p(n+\alpha)-\nu_{n}}} \\
\times \frac{\sqrt{n+\alpha}}{\sqrt{\left(p(n+\alpha)+\nu_{n}\right)\left(n+\alpha-p(n+\alpha)-\nu_{n}\right)}} .
\end{gathered}
$$

Note that

$$
\frac{(n+\alpha)}{\left(p(n+\alpha)+\nu_{n}\right)\left(n+\alpha-p(n+\alpha)-\nu_{n}\right)}=\frac{(n+\alpha)}{(n+\alpha)^{2}\left(p+\frac{\nu_{n}}{n+\alpha}\right)\left(1-\left(p+\frac{\nu_{n}}{n+\alpha}\right)\right)} .
$$

Set $a=p+\nu_{n} /(n+\alpha)$ and define a function $f$ by $f(a)=a(1-a)$. Then $f(a)$ has a minimum value of $1 / 4$ at $a=1 / 2$. Therefore $1 / \sqrt{f(a)} \leq 2$. From (11)

$$
\begin{aligned}
& \left(\begin{array}{c}
n+\alpha \\
k_{n}
\end{array}\right) \leq 2 \frac{1}{\left(p+\frac{\nu_{n}}{n+\alpha}\right)^{p(n+\alpha)+\nu_{n}}} \times \frac{1}{\left(1-p-\frac{\nu_{n}}{n+\alpha}\right)^{(1-p)(n+\alpha)-\nu_{n}}} \times \frac{1}{\sqrt{n+\alpha}} \\
& =2 \frac{1}{p^{p(n+\alpha)+\nu_{n}}\left(1+\frac{\nu_{n}}{p(n+\alpha)}\right)^{p(n+\alpha)+\nu_{n}}} \times \frac{1}{(1-p)^{(1-p)(n+\alpha)-\nu_{n}}\left(1-\frac{\nu_{n}}{(1-p)(n+\alpha)}\right)^{(1-p)(n+\alpha)-\nu_{n}}} \\
& \quad \times \frac{1}{\sqrt{n+\alpha}} .
\end{aligned}
$$

Since $(1-p) / p=(1 / p)-1$ and $p$ is a fixed positive constant between 0 and $1,(p /(1-p))^{-\nu_{n}}$ is clearly bounded. So also is $\left(1+\nu_{n} /(p(n+\alpha))\right)^{-p(n+\alpha)-\nu_{n}}$.

Let $g(p)=1-\nu_{n} /(1-p)(n+\alpha)$. Then

$$
g^{\prime}(p)=\frac{\nu_{n}}{(1-p)^{2}(n+\alpha)},
$$


F. Aydin Akgun, B. E. Rhoades / Eur. J. Pure Appl. Math, 13 (3) (2020), 390-402

and $g$ is decreasing in $p$. Since $0<p<1$ and fixed, $g(p)$ is bounded, Using the above facts,

$\left(\frac{1-p}{p}\right)^{-\nu_{n}}$ and $\left(\frac{p}{1-p}\right)^{-\nu_{n}}\left(1+\frac{\nu_{n}}{p(n+\alpha)}\right)^{-(p(n+\alpha))+\nu_{n}}\left(1-\frac{\nu_{n}}{(1-p) n+\alpha}\right)^{-\left((1-p)(n+\alpha)+\nu_{n}\right)}$

are bounded. Also,

$$
\left(\begin{array}{c}
n+\alpha \\
k_{n}
\end{array}\right) \leq C_{3} \frac{1}{p^{p(n+\alpha)}} \times \frac{1}{(1-p)^{(1-p)(n+\alpha)}} \times \frac{1}{\sqrt{n+\alpha}} .
$$

We can write (12) as

$$
\left(\begin{array}{c}
n+\alpha \\
k_{n}
\end{array}\right) \leq C_{3} \frac{1}{\frac{q}{1+q}\left(\frac{q}{1+q}\right)(n+\alpha)} \times \frac{1}{\left(\frac{1}{1+q}\right)^{\left(\frac{1}{1+q}\right)(n+\alpha)}} \times \frac{1}{\sqrt{n+\alpha}} .
$$

From (13)

$$
\begin{aligned}
\left(\begin{array}{c}
n+\alpha \\
k_{n}
\end{array}\right)^{q^{k_{n}}} & \leq C_{3} \frac{1}{\left(\frac{q}{1+q}\right)^{\left(\frac{q}{1+q}\right)(n+\alpha)}} \times \frac{1}{\left(\frac{1}{1+q}\right)^{\left(\frac{1}{1+q}\right)(n+\alpha)}} \times \frac{1}{\sqrt{n+\alpha}} q^{k_{n}} \\
& =C_{3} \frac{1}{\left(\frac{q}{1+q}\right)^{\left(\frac{q}{1+q}\right)(n+\alpha)}} \times \frac{1}{\left(\frac{1}{1+q}\right)^{\left(\frac{1}{1+q}\right)(n+\alpha)}} \times \frac{1}{\sqrt{n+\alpha}} q^{\left(\frac{1}{1+q}\right)(n+\alpha)+\nu_{n}} \\
& =C_{3} \frac{(1+q)^{n+\alpha}}{\sqrt{n+\alpha}} q^{\nu_{n}} \leq C_{q} \frac{(1+q)^{n+\alpha}}{\sqrt{n+\alpha}} .
\end{aligned}
$$

Thus

$$
\max _{0 \leq k \leq n}\left(\begin{array}{l}
n+\alpha \\
k+\alpha
\end{array}\right) q^{k+\alpha} \leq C_{q} \frac{(1+q)^{n+\alpha}}{\sqrt{n+\alpha}}
$$

From (9)

$$
\begin{aligned}
& K^{k} \sum_{r=0}^{\infty} \sum_{n=2^{r}+1}^{2^{r+1}} \gamma(n)^{k} n^{k-1}\left\{\sum_{m=0}^{n}\left(\begin{array}{c}
n+\alpha \\
m+\alpha
\end{array}\right)^{2}\left(\frac{m+\alpha}{n+\alpha}\right)^{2} q^{2 n-2 m}(1+q)^{-2 n-2 \alpha}\left|b_{m}\right|^{2}\right\}^{k / 2} \\
= & K^{k} \sum_{r=0}^{\infty} \sum_{n=2^{r}+1}^{2^{r+1}} \gamma(n)^{k} n^{k-1}\left\{\sum_{m=0}^{n}\left(\begin{array}{c}
n+\alpha \\
m+\alpha
\end{array}\right)\left(\frac{m+\alpha}{n+\alpha}\right)^{2}\left(\begin{array}{c}
n+\alpha \\
n-m
\end{array}\right) q^{n-m} q^{n-m}(1+q)^{-2 n-2 \alpha}\left|b_{m}\right|^{2}\right\}^{k / 2} .
\end{aligned}
$$

Using Lemma 4, equation (14) can be written as

$\leq K^{k} C_{q}^{k / 2} \sum_{r=0}^{\infty} \sum_{n=2^{r}+1}^{2^{r+1}} \gamma(n)^{k} n^{k-1}\left\{\sum_{m=0}^{n}\left(\begin{array}{c}n+\alpha \\ m+\alpha\end{array}\right)\left(\frac{m+\alpha}{n+\alpha}\right)^{2} \frac{1}{\sqrt{n+\alpha}} q^{n-m}(1+q)^{-2 n-2 \alpha}\left|b_{m}\right|^{2}\right\}^{k / 2}$ 


$$
=K^{k} C_{q}^{k / 2} \sum_{r=0}^{\infty} \sum_{n=2^{r}+1}^{2^{r+1}} \gamma(n)^{k} n^{k-1}\left\{\sum_{m=0}^{n}\left(\begin{array}{c}
n+\alpha \\
m+\alpha
\end{array}\right)(m+\alpha)^{2} \frac{1}{(n+\alpha)^{5 / 2}} q^{n-m}(1+q)^{-2 n-2 \alpha}\left|b_{m}\right|^{2}\right\}^{k / 2} .
$$

For $k=2$, the above inequality becomes

$$
\Omega \leq K^{2} C_{q} \sum_{r=0}^{\infty} \sum_{n=2^{r}+1}^{2^{r+1}} \gamma(n)^{2} n(n+\alpha)^{-5 / 2}\left\{\sum_{m=0}^{n}\left(\begin{array}{c}
n+\alpha \\
m+\alpha
\end{array}\right)(m+\alpha)^{2} q^{n-m}(1+q)^{-2 n-2 \alpha}\left|b_{m}\right|^{2}\right\}^{5 / 2} .
$$

Lemma 5. There exists a $D_{q}>0$ such that

$$
\sum_{n=m}^{\infty}\left(\begin{array}{c}
n+\alpha \\
m+\alpha
\end{array}\right) q^{n-m}(1+q)^{-n-\alpha} \leq D_{q}
$$

for all $m \in \mathbb{Z}^{+}$and $1 \leq k \leq 2$.

Proof. The proof of the lemma is easy to verify and it is a generalization of Theorem B of [6] to E-J matrices. Using Theorem B of [6], we can write (16) as

$$
\begin{aligned}
\Omega & \leq K^{2} C_{q} D_{q} \sum_{r=0}^{\infty} \gamma\left(2^{r+1}\right)^{2} 2^{r}\left(2^{r}+\alpha\right)^{-5 / 2} \sum_{m=0}^{2^{r+1}}(m+\alpha)^{2}\left|b_{m}\right|^{2} \\
& \leq K^{2} C_{q} D_{q} \sum_{r=0}^{\infty} \gamma\left(2^{r+1}\right)^{2}\left(2^{r}+\alpha\right)^{-3 / 2} \sum_{m=0}^{2^{r+1}}(m+\alpha)^{2}\left|b_{m}\right|^{2}, \quad \alpha>0 .
\end{aligned}
$$

Let $p=2 / k$. By Hölder's inequality, for $\alpha>0$ and $1 \leq k \leq 2$,

$\Omega=K^{k} C_{q}^{k / 2} \sum_{r=0}^{\infty}\left(\sum_{n=2^{r}+1}^{2^{r+1}} \gamma(n)^{k q} n^{q\left(\frac{-k}{4}-1\right)}\right)^{1 / q}\left\{\sum_{n=2^{r}+1}^{2^{r+1}} \sum_{m=0}^{n}\left(\begin{array}{c}n+\alpha \\ m+\alpha\end{array}\right)(m+\alpha)^{2} q^{n-m}(1+q)^{-n-\alpha}\left|b_{m}\right|^{2}\right\}$.

Since $\gamma \in \Gamma(\beta)$ and, for $\beta \in \mathbb{R}$, by Theorem $\mathrm{A}$ in [6], we can write the expression in the first bracket in (17) as

$$
\begin{aligned}
\left(\sum_{n=2^{r}+1}^{2^{r+1}} \gamma(n)^{k q} n^{q\left(\frac{-k}{4}-1\right)}\right)^{1 / q} & \leq K^{1 / q} \gamma\left(2^{r}+1\right)^{k}\left(2^{r}+1\right)^{\left(-\frac{k}{4}-1\right)} \\
& \leq K^{1 / q} \gamma\left(2^{r}+1\right)^{k}\left(2^{r}\right)^{\left(-\frac{k}{4}-1\right)}
\end{aligned}
$$

Thus, from (17),

$\Omega \leq K^{k+\frac{1}{q}} C_{q}^{k / 2} \sum_{r=0}^{\infty} \gamma\left(2^{r+1}\right)^{k}\left(2^{r}\right)^{\left(\frac{-k}{4}-1\right)}\left\{\sum_{n=2^{r}+1}^{2^{r+1}} \sum_{m=0}^{n}\left(\begin{array}{c}n+\alpha \\ m+\alpha\end{array}\right)(m+\alpha)^{2} q^{n-m}(1+q)^{-n-\alpha}\left|b_{m}\right|^{2}\right\}^{k / 2}$. 
F. Aydin Akgun, B. E. Rhoades / Eur. J. Pure Appl. Math, 13 (3) (2020), 390-402

Changing the order of summation inside the brackets in the above inequality, (18) is equal to

$$
\begin{aligned}
& =K^{k+\frac{1}{q}} C_{q}^{k / 2} \sum_{r=0}^{\infty}\left\{\gamma\left(2^{r+1}\right)^{k q}\left(2^{r}\right)^{q\left(-\frac{k}{4}-1\right)}\right\}^{\frac{1}{q}}\left\{\sum_{m=0}^{2^{r+1}} \sum_{n=2^{r}+1}^{2^{r+1}}\left(\begin{array}{c}
n+\alpha \\
m+\alpha
\end{array}\right)(m+\alpha)^{2} q^{n-m}(1+q)^{-n-\alpha}\left|b_{m}\right|^{2}\right\}^{k / 2} \\
& \leq K^{k+\frac{1}{q}} C_{q}^{k / 2} \sum_{r=0}^{\infty} \gamma\left(2^{r+1}\right)^{k}\left(2^{r}\right)^{\left(-\frac{k}{4}-1\right)}\left\{\sum_{m=0}^{2^{r}+1} \sum_{n=2^{r}+1}^{2^{r+1}}\left(\begin{array}{c}
n+\alpha \\
m+\alpha
\end{array}\right)(m+\alpha)^{2} q^{n-m}(1+q)^{-n-\alpha}\left|b_{m}\right|^{2}\right\}^{k / 2} \\
& +K^{k+\frac{1}{q}} C_{q}^{k / 2} \sum_{r=0}^{\infty} \gamma\left(2^{r+1}\right)^{k}\left(2^{r}\right)^{\left(-\frac{k}{4}-1\right)}\left\{\sum_{m=2^{r}+1}^{2^{r+1}} \sum_{n=2^{r}+1}^{2^{r+1}}\left(\begin{array}{c}
n+\alpha \\
m+\alpha
\end{array}\right)(m+\alpha)^{2} q^{n-m}(1+q)^{-n-\alpha}\left|b_{m}\right|^{2}\right\}^{k / 2} .
\end{aligned}
$$

Using Lemma 5,

$$
\begin{aligned}
\Omega & \leq K^{k+\frac{1}{q}} C_{q}^{k / 2} \sum_{r=0}^{\infty} \gamma\left(2^{r+1}\right)^{k}\left(2^{r}\right)^{\left(-\frac{k}{4}-1\right)}\left\{\sum_{m=0}^{2^{r}+1} D_{q}(m+\alpha)^{2}\left|b_{m}\right|^{2}\right\}^{k / 2} \\
& +K^{k+\frac{1}{q}} C_{q}^{k / 2} \sum_{r=0}^{\infty} \gamma\left(2^{r+1}\right)^{k}\left(2^{r}\right)^{\left(-\frac{k}{4}-1\right)}\left\{\sum_{m=2^{r}+1}^{2^{r+1}} D_{q}(m+\alpha)^{2}\left|b_{m}\right|^{2}\right\}^{k / 2} \\
& \leq K^{k+\frac{1}{q}} C_{q}^{k / 2} \sum_{r=0}^{\infty} \gamma\left(2^{r+1}\right)^{k}\left(2^{r}\right)^{\left(-\frac{k}{4}-1\right)}\left\{\sum_{m=0}^{2^{r+1}} D_{q}(m+\alpha)^{2}\left|b_{m}\right|^{2}\right\}^{k / 2} \\
& \leq K^{k+\frac{1}{q}} C_{q}^{k / 2} \sum_{r=0}^{\infty} \gamma\left(2^{r+1}\right)^{k}\left(2^{r}\right)^{\left(-\frac{3 k}{4}\right)}\left\{\sum_{m=0}^{2^{r+1}} D_{q}(m+\alpha)^{2}\left|b_{m}\right|^{2}\right\}^{k / 2} \\
& \leq K^{k+\frac{1}{q}} C_{q}^{k / 2} D_{q}^{k / 2} \sum_{r=0}^{\infty} \gamma\left(2^{r+1}\right)^{k}(2 r)^{\left(-\frac{3 k}{4}\right)}\left\{\sum_{m=0}^{2^{r+1}}(m+\alpha)^{2}\left|b_{m}\right|^{2}\right\}^{k / 2} .
\end{aligned}
$$

For $1 \leq k \leq 2$,

$$
\Omega \leq L \sum_{r=0}^{\infty} \gamma\left(2^{r+1}\right)^{k}\left(2^{r}\right)^{\left(-\frac{3 k}{4}\right)}\left\{\sum_{m=0}^{2^{r+1}}(m+\alpha)^{2}\left|b_{m}\right|^{2}\right\}^{k / 2},
$$

where $L=K^{k+\frac{1}{q}} C_{q}^{k / 2} D_{q}^{k / 2}$. From (19),

$\Omega \leq L \sum_{r=0}^{\infty} \gamma\left(2^{r+1}\right)^{k}\left(2^{r}\right)^{\left(-\frac{3 k}{4}\right)}\left\{\alpha^{2}\left|b_{0}\right|^{2}+\sum_{s=0}^{r} \sum_{m=2^{s}+1}^{2^{s+1}}(m+\alpha)^{2}\left|b_{m}\right|^{2}\right\}^{k / 2}$ 


$$
\leq L \sum_{s=0}^{\infty} \sum_{r=s}^{\infty} \gamma\left(2^{r+1}\right)^{k}\left(2^{r}\right)^{\left(-\frac{3 k}{4}\right)}\left\{\sum_{m=2^{s}+1}^{2^{s+1}}(m+\alpha)^{2}\left|b_{m}\right|^{2}\right\}^{k / 2}+L \sum_{r=0}^{\infty} \gamma\left(2^{r+1}\right)^{k}\left(2^{r}\right)^{\left(-\frac{3 k}{4}\right)} \alpha^{k}\left|b_{0}\right|^{k} .
$$

Here $\{\gamma(n)\}$ is quasi $\beta$-power monotone decreasing and $\left\{n^{-3 / 4} \gamma(n)\right\}$ is quasi $\epsilon$-power monotone decreasing, where $\beta>3 / 4$ and $\epsilon=\beta+3 / 4$. Thus, by using Lemma 1 of [6],

$$
\sum_{n=m}^{\infty} \gamma\left(2^{n}\right)^{k}\left(2^{n}\right)^{-3 k / 4} \leq M \gamma\left(2^{m}\right)^{k}\left(2^{m}\right)^{-3 k / 4}, M \in \mathbb{Z}^{+} .
$$

Therefore

$$
\begin{aligned}
\Omega & \leq L \sum_{s=0}^{\infty} \gamma\left(2^{s+1}\right)^{k}\left(2^{s}\right)^{(-3 k / 4)}\left\{\sum_{m=2^{s}+1}^{2^{s+1}}(m+\alpha)^{2}\left|b_{m}\right|^{2}\right\}^{k / 2}+L \gamma(2)^{k} \alpha^{k}\left|b_{0}\right|^{k} \\
& \leq 2^{k} L \sum_{s=0}^{\infty} \gamma\left(2^{s}\right)^{k}\left(2^{s}\right)^{(-3 k / 4)}\left\{\sum_{m=2^{s}+1}^{2^{s+1}}(m+\alpha)^{2}\left|b_{m}\right|^{2}\right\}^{k / 2}+L \gamma(2)^{k} \alpha^{k}\left|b_{0}\right|^{k} \\
& \leq 2^{k} L \sum_{s=0}^{\infty} \gamma\left(2^{s}\right)^{k}\left\{\sum_{m=2^{s}+1}^{2^{s+1}}(m+\alpha)^{2}\left|b_{m}\right|^{2}\right\}^{k / 2}+L \gamma(2)^{k} \alpha^{k}\left|b_{0}\right|^{k}
\end{aligned}
$$

and thus

$$
\begin{aligned}
& \sum_{n=2}^{\infty} \gamma(n)^{k} n^{k-1} \int_{0}^{1}\left|\sigma_{n}(x)-\sigma_{n-1}(x)\right|^{k} d x \\
& \leq 2^{\left(-\frac{3 k}{4}\right)} L \sum_{s=0}^{\infty} \gamma\left(2^{s}\right)^{k}\left\{\sum_{m=2^{s}+1}^{2^{s+1}}(m+\alpha)^{2}\left|b_{m}\right|^{2}\right\}^{k / 2}+L \gamma(2)^{k} \alpha^{k}\left|b_{0}\right|^{k} .
\end{aligned}
$$

The following Corollaries can be verified by taking $\alpha=0$ in the above theorems.

Corollary 1. Every orthogonal series $\sum_{n=0}^{\infty} c_{n} \psi_{n}, c_{n} \in \ell_{2}\left(\mathbb{Z}^{+}\right)$is $|H, \psi|_{k}$ summable for $1 \leq k \leq 2$ and $\gamma \in \Gamma_{\beta}$ with $\beta>1-l / k$, where $\left\{\psi_{n}\right\}_{n=0}^{\infty} \subset L_{2}[0,1]$ and $H$ is a Hausdorff matrix with entries $\left(h_{n k}\right)_{n, k} \in \mathbb{Z}^{+}$.

This is Theorem 2 of $[6]$.

Corollary 2. Let $1 \leq k \leq 2$ and $\gamma \in \Gamma_{\beta}$ with $\beta>-3 / 4$, where $\left\{\phi_{n}\right\}_{n=0}^{\infty} \subset L_{2}[0,1]$ and $H$ is a Hausdorff matrix. Then, for any $c_{n} \in \ell^{2}\left(\mathbb{Z}^{+}\right)$, a sufficient condition for the orthogonal series $\sum_{n=0}^{\infty} c_{n} \psi_{n}$ to be $|H, \gamma|_{k}$ summable is

$$
\sum_{m=0}^{\infty} \gamma\left(2^{m}\right)^{k}\left\{\sum_{n=2^{m}+1}^{2^{m+1}} \sqrt{n}\left|c_{n}\right|^{2}\right\}^{k / 2}<\infty
$$

This includes the results of Theorem 3 of [6]. 


\section{References}

[1] K Endl. Abstracts of short communications and scientific program. Int. Congress of Math., page 46, 1960.

[2] F Hausdorff. Summationmethoden und momentfolgen i.. Math. Z., 9:74-109, 1921.

[3] F Hausdorff. Summationmethoden und momentfolgen ii.. Math. Z., 9:280-299, 1921.

[4] W A Hurwitz and L L Silverman. On the consistency and equivalence of certain definitions of summability. Trans. Amer. Math. Soc., 18:1-20, 1917.

[5] A Jakimovski. The product of summability methods; new classes of transformations and their properties, i,ii.. Contract No. AF61(052)-187, Technical (Scientific) Note No. 2,1959 .

[6] K Kalaivana and G P Youvaraj. Generalized absolute Hausdorff summability of orthogonal series. Acta. Math Hungarica, 140:169-186, 2013.

[7] O A Ziza. On the summation of orthogonal series by Euler's method. Matem. Sb., 66:354-377, 1965. 